\title{
BMJ Open Early screening for post-stroke depression, and the effect on functional outcomes, quality of life and mortality: a protocol for a systematic review and meta-analysis
}

\author{
Sudhakar Selvaraj, ${ }^{1,2}$ Teresa Arora, ${ }^{3}$ Tahani Casameni Montiel, ${ }^{4}$ Ian Grey, ${ }^{5}$ \\ Hind Alfraih, ${ }^{6}$ Melissa Fadipe, ${ }^{7}$ Robert Suchting, ${ }^{1}$ Sean Savitz, ${ }^{2,8}$ \\ Jennifer E Sanner Beauchamp, ${ }^{2,7}$ Linda Östlundh (1) ${ }^{9}$
}

To cite: Selvaraj S, Arora T, Casameni Montiel T, et al. Early screening for post-stroke depression, and the effect on functional outcomes, quality of life and mortality: a protocol for a systematic review and meta-analysis. BMJ Open 2021;11:e050451. doi:10.1136/ bmjopen-2021-050451

- Prepublication history and additional supplemental material for this paper are available online. To view these files, please visit the journal online. (http://dx.doi.org/10.1136/ bmjopen-2021-050451).

SSe and TA contributed equally.

SSe and TA are joint first authors.

Received 19 February 2021 Accepted 21 July 2021

Check for updates

(c) Author(s) (or their employer(s)) 2021. Re-use permitted under CC BY-NC. No commercial re-use. See rights and permissions. Published by BMJ.

For numbered affiliations see end of article.

Correspondence to

Ms. Linda Östlundh;

lostlundh@uaeu.ac.ae

\section{ABSTRACT}

Introduction Post-stroke depression (PSD) is a severe complication of cerebrovascular stroke affecting about one-third of stroke survivors. Moreover, PSD is associated with functional recovery and quality of life (QOL) in stroke survivors. Screening for PSD is recommended. There are, however, differences in the literature on the impact of early screening on functional outcomes. In this systematic review, we synthesise the currently available literature regarding the associations between timing and setting of PSD screening and mortality, QOL and functional outcomes in stroke survivors.

Methods and analysis We will systematically search electronic databases including PubMed, Embase, APA PsycINFO, Web of Science, Scopus and CINAHL from inception to August 2021. Four reviewers will screen the title and abstract and full-text level records identified in the search in a blinded fashion to determine the study eligibility. Any selection disagreements between the reviewers will be resolved by the study investigator. Data extraction of eligible studies will be conducted by two reviewers using a predefined template. We will complete the quality assessment of included articles independently by two reviewers using the Newcastle Ottawa Scale. Eventual discrepancies will be resolved by the principal investigator. Ethics and dissemination Due to the nature of the study design, ethical approval is not required. The systematic review and meta-analysis findings will be published and disseminated in a peer-reviewed journal. Our results will also be disseminated through posters and presentations at appropriate scientific conferences.

PROSPERO registration number CRD42021235993.

\section{INTRODUCTION}

Post-stroke depression (PSD) is characterised by depressed or dysphoric mood, reduced motivation, energy and libido, as well as sleep disorders. It is a severe complication of cerebrovascular stroke ${ }^{1}$ affecting about one-third of stroke survivors. ${ }^{23}$ PSD appears to be the most common psychiatric sequelae
Strengths and limitations of this study

- Comprehensive systematic review of the associations between post-stroke depression and stroke outcomes.

- Rigorous methods, following the Preferred Reporting Items for Systematic Reviews and Meta-Analyses guidelines, will be conducted to minimise the risk of bias.

- Limitations of this review include the exclusion of papers in languages other than English and unpublished, grey materials.

of stroke, and is independently associated with increased morbidity, mortality and disability. ${ }^{4-6}$ PSD is also considered one of the most significant predictors for functional recovery and quality of life (QOL) in stroke survivors. ${ }^{78}$ Stroke survivors with PSD are more likely to commit suicide than patients without PSD. ${ }^{9}{ }^{10}$ Suicide rates are more than double in stroke survivors compared with the general population. ${ }^{11}$ Some stroke survivors who subsequently developed depression, and had a previous history of depression, reported suicidal thoughts as early as 4 days after the onset of stroke. ${ }^{12}$ Disability or dependence in activities of daily living (ADLs) of patients who have suffered a stroke may also increase the risk of $\mathrm{PSD}^{13}$ and suicidal ideation. This highlights an important, yet vicious cycle between PSD and functional disabilities. Therefore, stroke survivors with functional disabilities should be considered at-risk for PSD and suicidal ideation. Thus, targeted screening for PSD and suicidal ideation is warranted.

PSD has been consistently associated with poorer functional outcomes ${ }^{13}$ (eg, inability 
to perform ADLs independently) and rehabilitation outcomes (eg, extended duration of inpatient rehabilitation). ${ }^{13-15}$ It has been hypothesised that poorer functional outcomes (mortality, limitation of daily activities, stroke recurrences, cognitive impairment) associated with PSD may be due to limited rehabilitation participation. ${ }^{13} 16$ For example, in a retrospective, case-control study of 560 ischaemic stroke survivors, those with PSD had worse mobility, longer rehabilitation length of stay and greater insufficient response to rehabilitation efforts (as measured by lower Barthel Index (BI) and Rivermead Mobility Index (RMI)) than stroke survivors without PSD. ${ }^{14}$ Furthermore, stroke survivors adequately treated for PSD with antidepressants showed improved functional outcomes compared with antidepressant therapy non-responders. ${ }^{14}$ However, uncertainty regarding optimal strategies for treating PSD remains (eg, timing, dosing, duration and pharmaceuticals). ${ }^{17}$ Notably, while an independent association exists between PSD and poor functional recovery ${ }^{18}$ severe functional disabilities after stroke are also related to PSD. ${ }^{19}$

The period of time immediately after a stroke event is critical. For example, studies have shown that a substantial functional recovery occurs within 3 months after the first onset of stroke, ${ }^{20}$ compared with the later chronic phase. ${ }^{21}$ Rehabilitation and training can improve recovery during the period immediately after focal brain damage. Moreover, these rehabilitation interventions are critical for the functional restoration of patients who had a stroke..$^{2022} \mathrm{PSD}$ is a key factor that can influence stroke recovery. Despite the high prevalence of PSD, and its associated risks, only a minority of stroke survivors are adequately screened for PSD. Despite this, optimal strategies (timing, setting and methods) for PSD screening are currently unclear. ${ }^{123}$ The risk of depression in stroke survivors is high immediately after the acute stroke, especially within the 3 months after a stroke, ${ }^{24}$ during which the prevalence is estimated to be $33 \%$. Interestingly, nearly half of those who had depression in the first 3 months of acute stroke recovered at 1 year. ${ }^{24}$ Routine screening for PSD in at-risk patients (eg, those with severe functional disabilities and a history of depression) in the early stroke recovery period has been shown to reduce the risk of PSD. ${ }^{13}$ However, other studies did not find supportive evidence for PSD screening and stroke outcomes. ${ }^{26}{ }^{26}$ Nevertheless, randomised controlled clinical trials show that screening and collaborative care for depression treatment, can improve functional outcomes in chronic medical conditions, including stroke. ${ }^{27}$ The American Heart Association/American Stroke Association recommends screening for PSD in patients after acute stroke, as well as further research into the effect of PSD on all outcomes, including QOL. ${ }^{1}$ To optimise the care for patients who had a stroke, appropriate processes should be established before screening occurs to assure correct PSD diagnosis, adequate treatment and routine follow-up. ${ }^{1}$

A meta-analysis revealed an increased risk of mortality in those with PSD, ${ }^{28}$ where the estimated HR was 1.59 .
However, there are differences in published studies on the relationship between PSD and stroke recurrences. Sibolt $e t a l^{29}$ found that PSD was associated with increased ischaemic stroke recurrence. Conversely, Ayerbe et $a \vec{l}^{30}$ reported that PSD at 3 months was not associated with higher risk of total stroke recurrence over a 5-year follow-up period. Another systematic review and metaanalysis found a significant relationship between PSD and poor functional outcomes. ${ }^{13}$ None of the available systematic reviews have specifically investigated symptoms of depression at the acute stroke period on all strokerelated outcomes. Collectively, it is not clear whether early depression screening at acute stages of stroke can impact stroke recurrences, QOL and/or mortality.

The purpose of our review protocol is to update and synthesise the currently available literature in order to quantify the degree to which early onset PSD symptoms are associated with mortality, QOL and functional outcomes in stroke survivors. The primary research aims of this systematic review and meta-analysis are as follows:

1. To investigate if early PSD symptoms in hospitalised patients immediately after a stroke is associated with worse stroke-related disability within 3 months after an acute stroke event.

2. To investigate if early PSD (at acute hospital admission or within 3 months) is associated with long-term (>1 year) stroke-related health outcomes (strokerelated disability, stroke recurrence, mortality, QOL).

We hypothesise that patients with early PSD at hospital or within 3 months, will have a substantial disability, poorer QOL and increased mortality.

\section{METHODS AND ANALYSIS}

This protocol follows the Preferred Reporting Items for Systematic Reviews and Meta-Analyses Protocol (PRISMA-P) guidelines. ${ }^{31}$ The PRISMA-P checklist can be found in the online supplemental material 1 . The final review will be developed in accordance with the 2020 PRISMA statement ${ }^{32}$ and will be informed by the Cochrane Handbook for Systematic Reviews of Interventions. ${ }^{33}$

\section{Eligibility criteria}

\section{Inclusion criteria}

Our predefined inclusion criteria, throughout the screening process, are as follows:

(1) Uses a validated depression scale for screening after a stroke diagnosis; (2) human subjects; (3) longitudinal/ prospective studies only; (4) English language only; (5) peer-reviewed, published articles only; (6) reports on a short and/or long-term outcome (QOL, functional recovery [motor/disability index], mortality, recurrent stroke/morbidity); (7) stroke diagnosis; (8) reports on the relationship between PSD and at least one outcome measure (see point 6); and (9) the depression measurement should be obtained at the time of stroke diagnosis or within 3 months of the diagnosis. We will check if the clinical diagnosis of stroke was made as per the WHO 
definition of stroke: 'rapidly developing clinical signs of focal (or global) disturbance of cerebral function, with symptoms lasting 24 hours or longer or leading to death, with no apparent cause other than of vascular origin'. Therefore we will exclude transient ischaemic attack and stroke symptoms caused by subdural haemorrhage, tumours, poisoning or trauma. ${ }^{34}$ We will include validated depression instruments ${ }^{35}$ including Diagnostic and Statistical Manual of Mental Disorders, International classification of Diseases and specific scales such as Center for Epidemiologic Studies Depression Scale, Patient Health Questionnaire, Hospital Anxiety and Depression Scale and Hamilton Depression Rating Scale, Beck Depression Inventory, Stroke Aphasia Depression Questionnaire, Aphasia Depression Rating Scale, Geriatric Depression Scale.

\section{Exclusion criteria}

Our predefined exclusion criteria, throughout the screening process, are as follows:

(1) Studies involving animals; (2) conference abstracts, posters or papers; (3) editorials/letter to the editor, unless it reports original research data that meets our predefined inclusion criteria; (4) patients with any primary psychiatric illness, except for anxiety/depression; (5) patients with dementia, epilepsy, cancer or multiple sclerosis; (6) patients with neurological or neurodegenerative conditions, except for stroke/neurovascular; (7) reviews of any kind; (8) questionnaire validation studies; (9) case studies; (10) randomised controlled trials (RCTs); (11) paediatric studies (samples that include patients under the age of 18 years); (12) studies assessing the effectiveness of medication; (13) protocol papers; (14) studies with retrospective design; (15) studies that have less than 1-year follow-up period; and (16) theses and dissertations.

\section{Information sources and search strategy}

A comprehensive search of peer-reviewed literature from six electronic databases will be conducted from their inception through to August 2021. The following medical and health sciences databases will be included: PubMed (NLM), Embase (Elsevier), APA PsycINFO (EBSCOhost), Scopus (Elsevier), Web of Science (Clarivate) and CINAHL (EBSCOhost). A preliminary search string to support the development of the research question and preset inclusion and exclusion criteria, was conducted in February-March 2021. The search strategy was developed by a medical librarian who is specialised in the conduct of systematic reviews (LÖ). PubMed and PubMed's Medical Subject Headings (MeSH) was used to systematically identify relevant search terms and synonyms for review and further suggestions by a subject specialist (SSe). The pre-search in PubMed will be adapted to, and performed in, all six selected databases. All keywords will be searched in a combination of the fields: 'title', 'abstract' and in 'MeSH'/ 'thesaurus'. We will limit the search to English papers only. No additional filters or geographical limitations will be used to ensure the best possible literature inclusion. Systematic hand screening of the reference lists of the included papers identified by the search will be conducted independently by two reviewers. A search $\log$, with search technical specifications, as well as results and notes for all databases included in the search, will be appended to the review to support the appraisal and reproduction of the search. The literature search will be repeated before completing the data extraction, and potential new studies published during the work process will be added to the result. The details of the preliminary search preformed in PubMed is available in the (online supplemental material 2).

\section{Data management}

The result from the database search will be uploaded to the systematic review software, Covidence. ${ }^{36}$ Automatic deduplication and screening will be conducted in the software. The finally selected references will be exported to a reference management software to enable manuscript preparation. Covidence will also support the update of the search before publishing by a re-upload and deduplication of the complete result from all databases. This will ensure blinded screening of all additional back-file and new records added since the initial search in May 2021.

\section{Selection of studies}

All unique records identified in the database search will be screened for eligibility against the predefined inclusion and exclusion criteria by four independent reviewers, as follows: TA and HA will conduct the initial title and abstract screening. TM and IG will screen the papers selected for full-text review. After each screening module, eventual conflicts identified by the software will be resolved by a fifth reviewer (SSe). All stages of the screening and selection processes in Covidence follow the PRISMA workflow. ${ }^{32}$ The screening processes and blinding will be predefined in the software settings based on the authors' responsibilities.

The search result, deduplication, screening and selection process, including reasons for study exclusion, will be documented in a PRISMA (2020) flow diagram. Cabells Predatory Reports ${ }^{37}$ will be consulted to verify that eventual open access papers selected are not published in possible predatory journals.

\section{Data extraction}

An extraction template will be used to extract relevant data from each of the included studies. The template will be developed by the team statistician (RS) and the principal investigator (SSe). The data extraction template will be piloted on five articles initially and adjusted, if necessary. Information about population characteristics, length of follow-up, depression scale used, time of screening for depression, stroke disability ratings, rehabilitation length of stay, mobility status as well as QOL scales and scores will be documented. The effect size/measure of association between PSD screening and the outcome variables of interest, along with $95 \%$ CIs will also be extracted. Two 
reviewers will extract the information independently and any discrepancies will be resolved by the primary study investigator (SSe).

\section{Quality assessment of individual studies}

Two reviewers will independently assess the quality of evidence and the risk of bias of all eligible studies using the Newcastle Ottawa Scale. Eventual disagreements between the reviewers will be discussed with the principal investigator (SSe) until a consensus is reached.

\section{Data analysis and synthesis}

The systematic review will identify and report the number of qualifying articles and provide an overall summary. Information surrounding the sample size, population and outcomes of interest will be compared across the studies. Articles that contain appropriate statistical information for meta-analysis will be assessed by the team statistician (RS). The meta-analysis will calculate a pooled prevalence ratio/OR/HR and $95 \%$ CI for each outcome variable of interest. Specific outcomes that will be evaluated in the present study will include stroke disability scales (BI, Modified Rankin Scale, RMI), QOL (short form 36; stroke-specific QOL, Euro-QOL) and mortality (dichotomous). The lower bound of studies to be included in any given meta-analysis is two. ${ }^{38}$ Analyses will, however, include as many studies that meet our predefined inclusion/exclusion criteria. Finally, we will perform metaregression to account for the influence of stroke severity (National Institutes of Health, Glasgow Coma Scale), type of stroke (ischaemic vs haemorrhagic) and length of follow-up (months).

To account for potential sources of heterogeneity, we will use random effects models in all analyses. Both a narrative and pooled prevalence will be reported. A forest plot will be used to provide a visual summary of the point estimate and $95 \%$ CI for each study and the overall pooled effect. Visual inspection of funnel plots, with trim and fill as well as Egger's test of asymmetry, will be used to identify sources of bias. Individual study influence will be evaluated via leave-one-out jackknife sensitivity analysis, whereby the overall pooled effect is calculated while omitting each study in turn.

Analyses will be conducted using the package metafor ${ }^{39}$ in the R Statistical Computing Environment ${ }^{40}$ by the team statistician (RS). We will assess confidence in estimates by evaluating sources of bias in the main patient outcomes. We will follow the Grading of Recommendations Assessment, Development, and Evaluation guidelines. ${ }^{40}$

\section{Strengths and limitations}

The strength of the protocol is the study design including the longitudinal cohort design to focus on early poststroke period. Our review will also employ comprehensive search strategies, compliance to PRISMA guidelines for systematic reviews and our protocol is also registered with the international database, PROSPERO. We also acknowledge the following limitations. First, we will not include grey literature sources. However, our comprehensive search strategy covers six major databases that will provide relevant, published, high-quality papers. Although RCTs and retrospective studies can provide additional insight, we decided not to include these in our review for the following reasons. First, when we evaluated our pilot searches, we found that the range of interventions studied in RCTs was extremely variable. Moreover, controlling for outcomes are difficult. Furthermore, RCTs are typically shorter time-framed which is not aligned with our aim of investigating the early depression symptoms and its impact on long-term stroke outcome(s). We excluded retrospective studies, due to bias associated with these types of studies including selection and recall bias. Furthermore, it is difficult to ascertain temporal relationships between depression and stroke outcomes with retrospective studies.

\section{Review status}

A preliminary search was performed in PubMed with the start in February 2021 and the result was updated in July 2021 (see online supplemental material 2). The review is set to start in May 2021.

\section{Potential amendments}

To avoid reporting bias of the review, we do not intend to modify the protocol. However, if necessary, any changes to the time frame or process of the review will be reported through updates in the online registered PROSPERO protocol.

\section{Patient and public involvement}

No patients or members of the public are involved in this study.

\section{ETHICS AND DISSEMINATION}

Our institutional ethics board policies exempts ethical approval for systematic reviews. The review results will be published in a peer-reviewed journal and disseminated through abstracts, posters and oral presentations at relevant scientific conferences.

\section{Author affiliations}

${ }^{1}$ Louis Faillace, MD, Department of Psychiatry, McGovern Medical School at the University of Texas Health Science Center at Houston, Houston, Texas, USA

${ }^{2}$ Institute for Stroke and Cerebrovascular Disease, University of Texas Health Science Center at Houston, Houston, Texas, USA

${ }^{3}$ College of Natural \& Health Sciences, Zayed University, Abu Dhabi, UAE ${ }^{4}$ Cizik School of Nursing, University of Texas Health Science Center at Houston, Houston, Texas, USA

${ }^{5}$ Department of Cognitive Science, United Arab Emirates University, Al Ain, UAE ${ }^{6}$ Department of Psychology, College of Natural \& Health Sciences, Zayed University, Abu Dhabi, UAE

${ }^{7}$ Department of Research, Cizik School of Nursing, University of Texas Health Science Center at Houston, Houston, Texas, USA

${ }^{8}$ Department of Neurology, McGovern Medical School at the University of Texas Health Science Center at Houston, Houston, Texas, USA

${ }^{9}$ National Medical Library, College of Medicine and Health Sciences, United Arab Emirates University, Al Ain, UAE 
Contributors The protocol draft was prepared by LÖ, TA and SSe. The search will be performed by LÖ and the title and abstract screening will be conducted by TA and HA. The full-text screening will be completed by TM and IG. Data extraction will be completed by TA and MF and overseen by the team statistician, RS. The data analytical strategy was designed by TA, RS and SSe. SSa, SSe and JESB conceptualised the research questions and the study design.

Funding The authors have not declared a specific grant for this research from any funding agency in the public, commercial or not-for-profit sectors.

Competing interests None declared.

Patient consent for publication Not required.

Provenance and peer review Not commissioned; externally peer reviewed.

Supplemental material This content has been supplied by the author(s). It has not been vetted by BMJ Publishing Group Limited (BMJ) and may not have been peer-reviewed. Any opinions or recommendations discussed are solely those of the author(s) and are not endorsed by BMJ. BMJ disclaims all liability and responsibility arising from any reliance placed on the content. Where the content includes any translated material, BMJ does not warrant the accuracy and reliability of the translations (including but not limited to local regulations, clinical guidelines, terminology, drug names and drug dosages), and is not responsible for any error and/or omissions arising from translation and adaptation or otherwise.

Open access This is an open access article distributed in accordance with the Creative Commons Attribution Non Commercial (CC BY-NC 4.0) license, which permits others to distribute, remix, adapt, build upon this work non-commercially, and license their derivative works on different terms, provided the original work is properly cited, appropriate credit is given, any changes made indicated, and the use is non-commercial. See: http://creativecommons.org/licenses/by-nc/4.0/.

\section{ORCID iD}

Linda Östlundh http://orcid.org/0000-0001-5091-604X

\section{REFERENCES}

1 Towfighi A, Ovbiagele B, El Husseini N, et al. Poststroke depression: a scientific statement for healthcare professionals from the American heart Association/American stroke association. Stroke 2017;48:e30-43.

2 Robinson RG, Price TR. Post-stroke depressive disorders: a followup study of 103 patients. Stroke 1982;13:635-41.

3 Ayerbe L, Ayis S, Wolfe CDA, et al. Natural history, predictors and outcomes of depression after stroke: systematic review and metaanalysis. Br J Psychiatry 2013;202:14-21.

4 Hadidi N, Treat-Jacobson DJ, Lindquist R. Poststroke depression and functional outcome: a critical review of literature. Heart Lung 2009;38:151-62.

5 Chemerinski E, Robinson RG, Kosier JT. Improved recovery in activities of daily living associated with remission of poststroke depression. Stroke 2001;32:113-7.

6 Singh RDS, Pandhi A, Alexandrov AV. Post stroke depression. Cerebrovascular Diseases: IntechOpen 2019.

7 Carod-Artal FJ, Egido JA. Quality of life after stroke: the importance of a good recovery. Cerebrovasc Dis 2009;27:204-14.

8 Carod-Artal J, Egido JA, González JL, et al. Quality of life among stroke survivors evaluated 1 year after stroke: experience of a stroke unit. Stroke 2000;31:2995-3000.

9 Eriksson M, Glader E-L, Norrving B, et al. Poststroke suicide attempts and completed suicides: a socioeconomic and nationwide perspective. Neurology 2015;84:1732-8.

10 Bartoli F, Pompili M, Lillia N, et al. Rates and correlates of suicidal ideation among stroke survivors: a meta-analysis. $J$ Neurol Neurosurg Psychiatry 2017;88:498-504.

11 Fuller-Thomson E, Tulipano MJ, Song M. The association between depression, suicidal ideation, and stroke in a population-based sample. Int J Stroke 2012;7:188-94.

12 Santos CO, Caeiro L, Ferro JM, et al. A study of suicidal thoughts in acute stroke patients. J Stroke Cerebrovasc Dis 2012;21:749-54.

13 Kutlubaev MA, Hackett ML. Part II: predictors of depression after stroke and impact of depression on stroke outcome: an updated systematic review of observational studies. Int $J$ Stroke 2014:9:1026-36.

14 Paolucci S, losa M, Coiro P, et al. Post-stroke depression increases disability more than $15 \%$ in ischemic stroke survivors: a case-control study. Front Neurol 2019;10:926.

15 van de Weg FB, Kuik DJ, Lankhorst GJ. Post-stroke depression and functional outcome: a cohort study investigating the influence of depression on functional recovery from stroke. Clin Rehabil 1999;13:268-72.

16 Subramanian SK, Chilingaryan G, Sveistrup H, et al. Depressive symptoms influence use of feedback for motor learning and recovery in chronic stroke. Restor Neurol Neurosci 2015;33:727-40.

17 Hackam DG, Mrkobrada M. Selective serotonin reuptake inhibitors and brain hemorrhage: a meta-analysis. Neurology 2012;79:1862-5.

18 Schmid AA, Kroenke K, Hendrie HC, et al. Poststroke depression and treatment effects on functional outcomes. Neurology 2011;76:1000-5.

19 Hackett ML, Köhler S, O'Brien JT, et al. Neuropsychiatric outcomes of stroke. Lancet Neurol 2014;13:525-34.

20 Prabhakaran S, Zarahn E, Riley C, et al. Inter-individual variability in the capacity for motor recovery after ischemic stroke. Neurorehabil Neural Repair 2008;22:64-71.

21 Zeiler SR, Krakauer JW. The interaction between training and plasticity in the poststroke brain. Curr Opin Neurol 2013;26:609-16.

22 Ward NS. Restoring brain function after stroke - bridging the gap between animals and humans. Nat Rev Neurol 2017:13:244-55.

23 Herrmann N, Seitz D, Fischer H, et al. Detection and treatment of post stroke depression: results from the registry of the Canadian stroke network. Int J Geriatr Psychiatry 2011;26:n/a-200.

24 Ayerbe L, Ayis S, Crichton S, et al. The natural history of depression up to 15 years after stroke: the South London stroke register. Stroke 2013;44:1105-10.

25 Dowrick C, Buchan I. Twelve month outcome of depression in general practice: does detection or disclosure make a difference? BMJ 1995;311:1274-6.

26 Williams JW, Mulrow CD, Kroenke K, et al. Case-Finding for depression in primary care: a randomized trial. Am J Med 1999;106:36-43.

27 Katon WJ, Lin EHB, Von Korff M, et al. Collaborative care for patients with depression and chronic illnesses. N Engl J Med 2010;363:2611-20.

28 Cai W, Mueller C, Li Y-J, et al. Post stroke depression and risk of stroke recurrence and mortality: a systematic review and metaanalysis. Ageing Res Rev 2019;50:102-9.

29 Sibolt G, Curtze S, Melkas S, et al. Poststroke dementia is associated with recurrent ischaemic stroke. J Neurol Neurosurg Psychiatry 2013;84:722-6.

30 Ayerbe L, Ayis S, Crichton S, et al. The long-term outcomes of depression up to 10 years after stroke; the South London stroke register. J Neurol Neurosurg Psychiatry 2014;85:514-21.

31 Moher D, Shamseer L, Clarke M, et al. Preferred reporting items for systematic review and meta-analysis protocols (PRISMA-P) 2015 statement. Syst Rev 2015;4:1.

32 Page MJ, McKenzie JE, Bossuyt PM. The prisma 2020 statement: an updated guideline for reporting systematic reviews. BMJ 2021;372:71.

33 Higgins JPT, Thomas J, Chandler J. Cochrane Handbook for systematic reviews of interventions. 2 edn. Chichester (UK): John Wiley \& Sons, 2019.

34 Meader N, Moe-Byrne T, Llewellyn A, et al. Screening for poststroke major depression: a meta-analysis of diagnostic validity studies. $J$ Neurol Neurosurg Psychiatry 2014;85:198-206.

35 Covidence. systematic review software [program]. Melbourne, Australia: Veritas Health Innovation, 2021.

36 Report CP. Cabells predatory report Beaumont. Texas, USA, 2021.

37 Valentine JC, Pigott TD, Rothstein HR. How many studies do you need?: a primer on statistical power for meta-analysis. J Educ Behav Stat 2010;35:215-47.

38 Viechtbauer W. Conducting Meta-Analyses in $R$ with the metafor Package. J Stat Softw 2010;36:1-48.

$39 \square \mathrm{R}$ Core Team. R: A language and environment for statistical computing. R Foundation for Statistical Computing [program]. Vienna, Austria, 2013.

40 Guyatt GH, Oxman AD, Vist G, et al. GRADE guidelines: 4. rating the quality of evidence--study limitations (risk of bias). J Clin Epidemiol 2011;64:407-15. 